\title{
Interconnecting loT Devices to Improve the QoL of Elderly People
}

\author{
Daniel Flores-Martin \\ University of Extremadura, Spain \\ Alejandro Pérez-Vereda \\ University of Málaga, Spain \\ Javier Berrocal \\ University of Extremadura, Spain \\ Carlos Canal \\ University of Málaga, Spain \\ Juan M. Murillo \\ University of Extremadura, Spain
}

\section{ABSTRACT}

The rate at which the Internet is growing is unstoppable due to the large number of connected smart devices. Manufacturers often develop specific protocols for their own devices that do not usually follow any standards. This hinders the interconnection and coordination of devices from different manufacturers, limiting the number of daily activities that can be supported. Some works are proposing different techniques to reduce this barrier and avoid the vendor lock-in issue. Nevertheless, this interconnection should also depends on the context. In this chapter, the authors propose a system to dynamically identify the interconnections required each specific situation depending on the context. This proposal has been tested in case studies focused in elderly people with the aim of automating their daily tasks and improving their quality of life. Further, in a world with an accelerated population aging, there is an increasing interest on developing solutions for the elderly living assistance through IoT systems.

Keywords:

Internet of Things (IoT): it is the concept of basically connecting any device to the Internet (and/or to each other). This includes everything from cellphones, coffee makers, washing machines, headphones, lamps, wearable devices.

Interconnection: a link between smart devices to exchange information between them.

Collaboration: cooperation between devices that are capable of exchanging information to achieve a common objective.

Elderly People: people being past middle age.

Situational-Context: paradigm composed of the virtual profiles of all entities (people or devices) involved in a situation to achieve the highest level of comfort for these entities. 
Semantic Web: extension of the World Wide Web that allows the sharing and reuse of information and the integration of data from different contents, applications and information systems.

Ontology: computer ontology refers to the interpretation of a group of ideas within a specific domain that allows the interrelationship between those ideas.

\section{INTRODUCTION}

The Internet of Things (IoT) is becoming more and more important. This is due to the large amount of smart devices that are being developed. Recent studies estimate that by 2020 there will be 20-30 billion devices connected to the Internet (Nordrum, 2016; IronPaper, 2015). These devices can be applied for a multitude of fields such as smart cities, agriculture, automotive or healthcare (Haluza \& Jungwirth, 2015). The purpose of these devices is to make people's lives easier by automating tasks or helping users to perform them. In healthcare, the IoT paradigm allows more personalized, collaborative and preventive care, where patients are able to monitor and manage their own health. Besides, the responsibility for healthcare is shared between patients and medical staff (Metcalf, Milliard, Gomez, \& Schwartz, 2016). These solutions are particularly interesting, mainly due to limited resources or difficult access to them if we consider the ageing of the population and the depopulation of rural areas.

IoT devices can perform simple tasks such as monitoring blood pressure or glucose level, performing periodic reminders, enhancing drug management or notifying of certain events. These tasks are useful but the real potential of IoT devices lies in the interconnection between them to collaborate and to perform more complex tasks. Due to the great heterogeneity of devices on the market, where there are many different manufacturers and devices, this interconnection is not easy. In order to achieve this interconnection, each manufacturer usually develops its own communication protocols. This means that there is no defined communication standard and the risk of vendor lock-in increases. This phenomenon conditions users to acquire devices from the same manufacturers in order to achieve total compatibility, also complicating the possible migration to another in the future (Roman, Zhou, \& Lopez, 2013), and even to set manually each device, something that can be tedious for people who do not have a certain technical knowledge.

Different works have promoted alternative methods to make IoT devices work with each other, such as specific frameworks, such as (Shrestha, Kubler, \& Främling, 2014), where a framework is developed to integrate specific domain applications into IoT, or (Kim et al., 2016), which presents interfaces and interconnection procedures based on oneM2M (Swetina, Lu, Jacobs, Ennesser, \& Song, 2014). The use of ontologies and the Semantic Web are also becoming very important to solve these interconnection problems (Szilagyi \& Wira, 2016). The main objective of the Semantic Web is to improve the Internet by extending interoperability between computer systems using smart agents and applications that seek information without human intervention (Barnaghi, Wang, Henson, \& Taylor, 2012). These works help to solve the problem of device interconnection, but it is not an easy task, because technological diversity of smart devices must be taken into account, as well as the correct handling of the context, which is not always considered.

Besides, the development of context-sensitive software has proved successful (Perera, Zaslavsky, Christen, \& Georgakopoulos, 2014). IoT devices are becoming intelligent thanks to the information gathered about the context in which they are located, from near people or other devices. In order to reduce the interaction among people and devices, this interconnection must be adapted to the context. These drawbacks can be addressed by developing software capable of adapting its behaviour to people's needs (Perera et al., 2014; Taivalsaari \& Mikkonen, 2017). In this way, several research areas can contribute to provide this adaptation, such us Context Oriented Programming (COP), Ambient Intelligent (AmI), Semantic Web, and Machine Learning (ML). Most of these paradigms allow us to define behaviours for different scenarios at design 
time, so the adaptation of the devices is limited to situations that developers have been able to identify, making it impossible to adapt them to other situations that may arise from the context.

The authors of this work have proposed the Situational-Context paradigm (Berrocal, Garcia-Alonso, Canal, \& Murillo, 2016), where the context is treated as a way of analyzing the conditions that exist at a particular time and place identifying people's needs. In this work, the authors present an architecture that models the Situational-Context focused on healthcare for the elderly people domain, although it can be used for another domain. In addition, it allows to obtain data from users' profile, stored in their smartphones, to use them so that IoT devices can adapt their behaviour to people's needs. This process is carried out dynamically and at runtime, due to the different possibilities that can occur in a context are innumerable. Thanks to this, IoT devices can adapt their behaviour to situations not previously predefined.

\section{MOTIVATIONS}

Nowadays, the interconnection of IoT devices to achieve a coordinated work is still a problem that prevents to exploit the full potential of IoT paradigm.

To show the impact of this problem, we are going to use a scenario based on ageing and rurality. In the rest of this chapter the proposed scenario will be used to show the benefits of our work.

Juan is a 74 years old man who lives in La Calera in the southeast of Caceres, in the Las Villuercas Mountains. This morning, Juan went for a walk with his friend Emilio. They both have their mobile phones with them. Since the village is running out of inhabitants and they are getting older, their sons and daughters always ask them not to go out without their mobile phones in case something happens to them. Juan and Emilio are not aware of this, but their mobile phones can do much more than receive calls from their children. In addition, these devices are recording where they walk, where are them, with whom they are and are detecting each other so that their phones now know they are in company (Figure 1).

Juan has returned home and it's time to take his medicine. It is notified by the electronic pill dispenser that was given to him last month. This pill dispenser has also detected that there are no pills left for the next day (Figure 2). It is very important that Juan does not stop his heart treatment. Although his smartphone made the electronic prescription request, they were unable to bring his medication. There seems to have been a mistake at the pharmacy in Guadalupe. Fortunately, Emilio takes the same medication as Juan and received it last week. Juan and Emilio have received a message telling them that tomorrow Emilio must give Juan two doses (Figure 3).

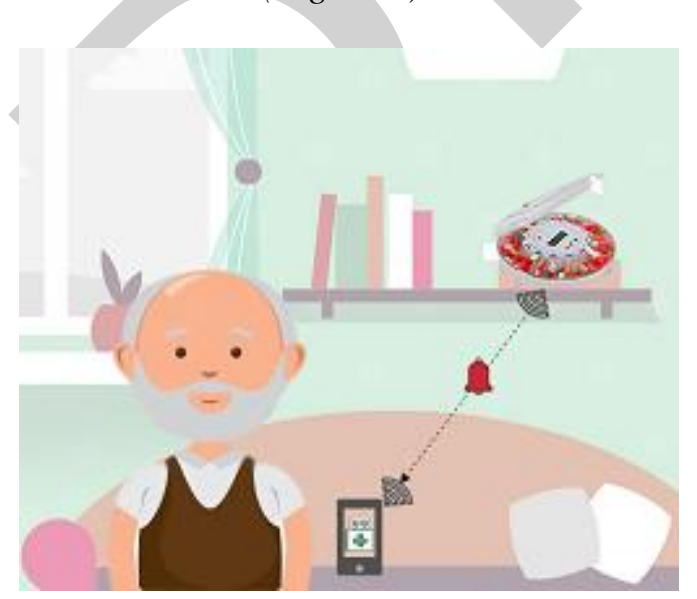

Figure 1. People Tracking

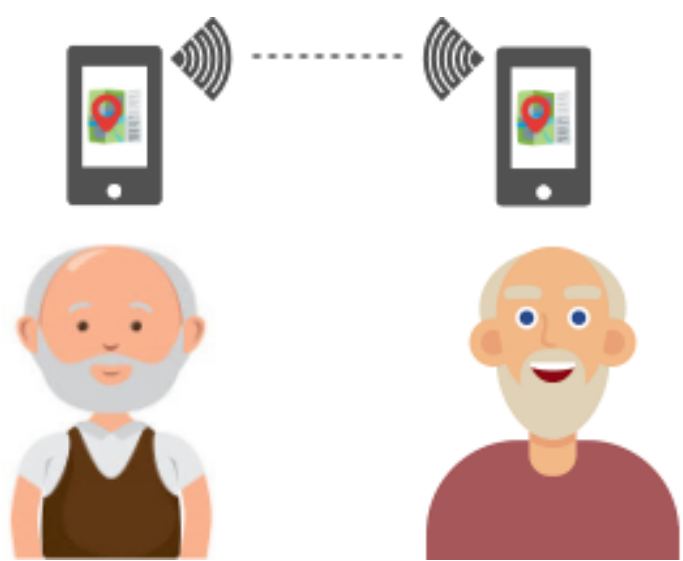

Figure 2. Pill Reminder 


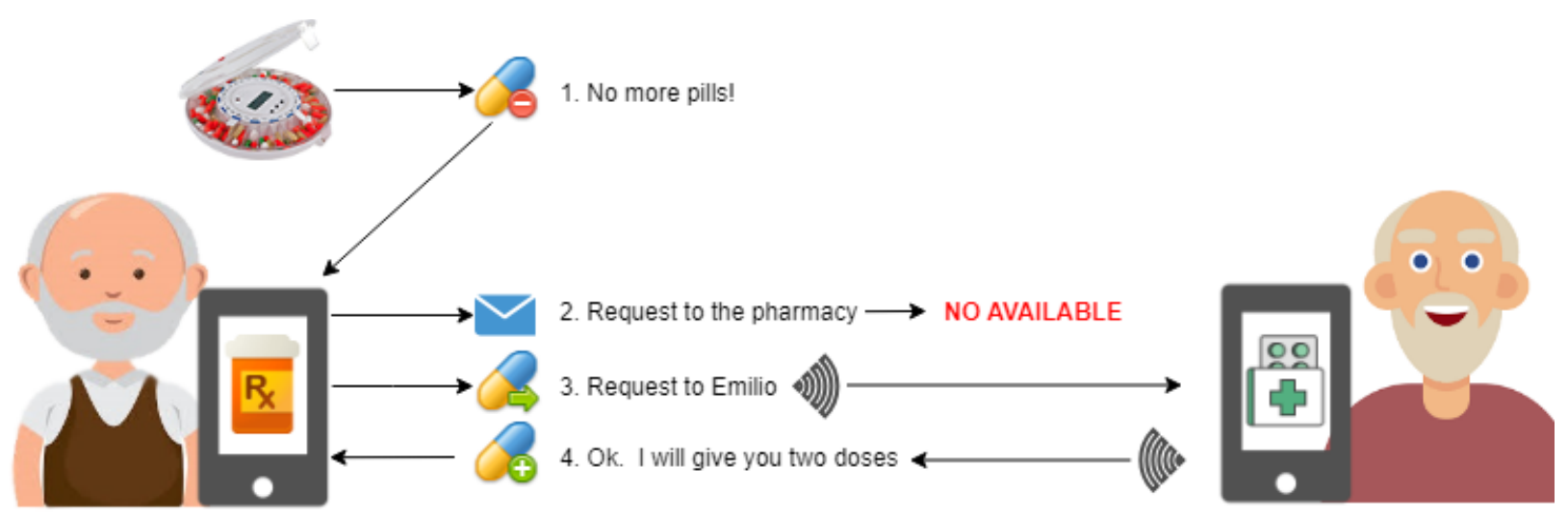

Figure 3. Doses Request Flow

The Semantic Web techniques and frameworks that have been seen before are not enough to solve this problem, since context information is needed.

This use case shows the need to interconnect different devices. In addition, this interconnection depends on the contextual information that surrounds the devices, so that all possible data can be had to carry out the interconnection in the best possible way. Therefore, we want to test the capacity of the Situational-Context to promote the generation of complex strategies that involve the orchestration of several devices.

\section{SITUATIONAL-CONTEXT}

Situational-Context is a way of analyzing the conditions that exist at a particular time and place in order to predict, at runtime, the expected IoT systems behaviour. It is composed of entities. These entities can be both IoT devices and people represented through their smartphones, indistinctly. In addition, they have two fundamental properties: skills and goals.

This model exploits the capabilities of smart devices to collect, store and calculate locally contextual information to build their virtual profile and the virtual profile of their owner. Therefore, the devices around it can reuse it to meet the user's preferences. Situational-Context defines that the virtual profile of an entity (IoT device or a person) must contain at least the following information:

- A Basic Profile that contains the raw contextual information dated with the status of the entity, the relationships with other devices and its history.

- A Social Profile. This profile contains the results of high-level inferences made on the Basic Profile.

- The Goals that details the state of the environment desired by the entity. These goals are deduced from the Basic and Social Profiles at runtime.

- The Skills that an entity has to make decisions and take actions capable of modifying the environment and aimed at achieving the goals.

Considering environments in which there are different entities and each of them has a virtual profile, Situational-Context can be defined as the composition of the virtual profiles of all entities involved in a particular situation.

Thanks to Situational-Context it is possible to analyze the context surrounding Juan and detect nearby entities, such as Emilio, or his smart pill dispenser, which can use its skill to make the electronic prescription when it detects that it is running out of doses of some type of pill, and thus be able to continue to provide Juan with his medication. In the same way, this could be applied to other entities such as Emilio (represented 
by his smartphone), Emilio's pill dispenser, or another IoT devices that they can have at home. SituationalContext aims, among others, to improve the QoL of people by avoiding having to invest so much time in configuring smart devices.

This paradigm has obtained previous results of interconnection of devices at the network level (GalánJiménez, Berrocal, Garcia-Alonso, Canal, \& Murillo, 2017). We assume, therefore, that the connection through the network of IoT devices in a Situational-Context environment is feasible.

\section{ENTITY REPRESENTATION ARCHITECTURE}

Due to the detected need, we use the Situational-Context paradigm with which we intend to achieve a better interconnection of IoT devices and get the maximum benefit by adapting their behaviour to people's preferences at runtime. This favors the interaction of entities and allows them to be dependent on the context in which they are, at runtime. Its components are detailed in Figure 4.

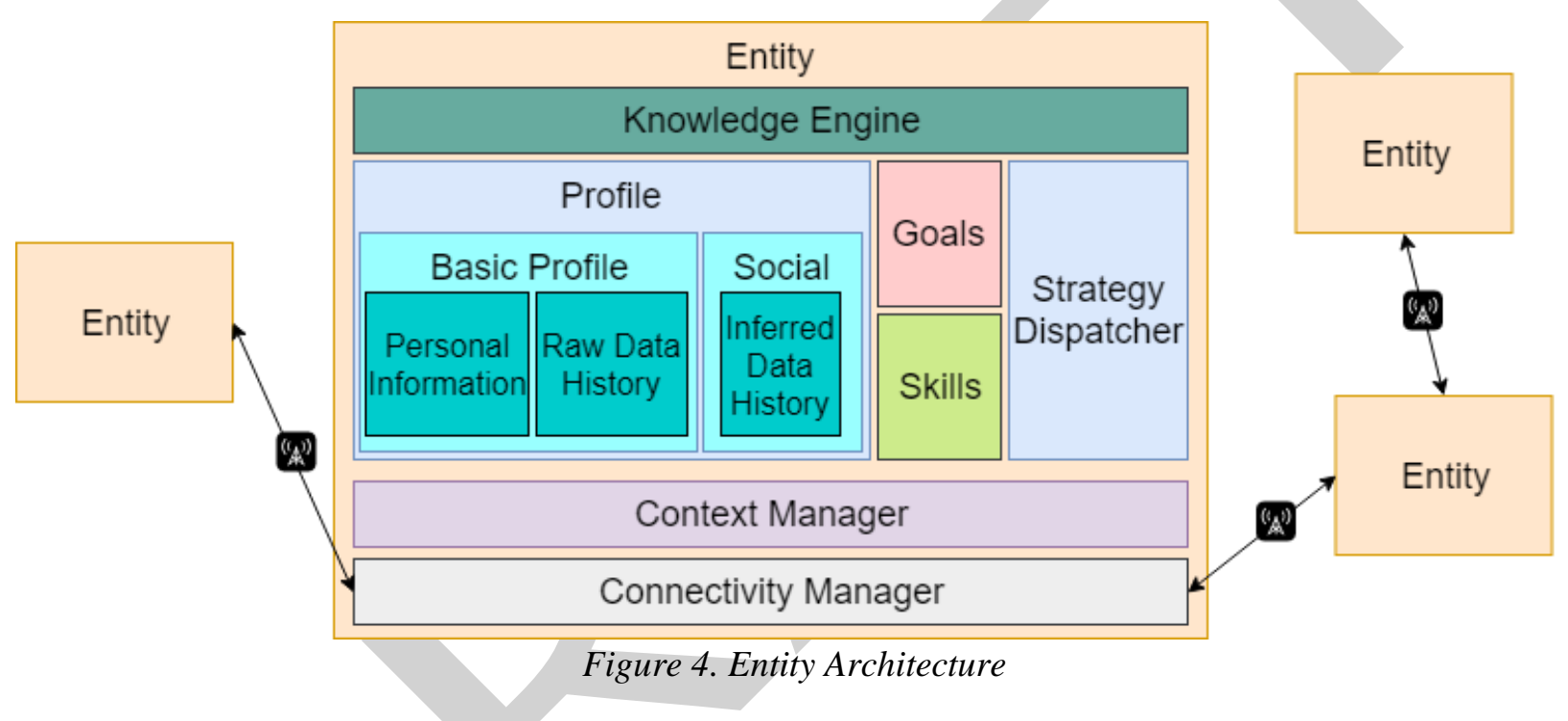

- Connectivity Manager. Establishes the physical connection between entities. It sends and receives information related to skills, goals, personal information, etc.

- Context Manager. Responsible for creating and updating contextual information. It contains the information of the entities belonging to the same situation in a given instant of time.

- Profile. Union of the Basic and the Social Profile of the entity.

- Basic Profile. Basic information that identifies the entity, such as the identifier, manufacturer, model, date of manufacture, etc. (Personal Information). It also contains raw data about the history of interactions with other entities (Raw Data History).

- Social Profile. Stores all inferred data from the basic profile (Inferred Data History). Thanks to this data, Juan's smartphone can know where Juan is moving or who he's accompanied by.

- Skills. Entity features. They produce a change in the context. For example, the pill dispenser can order pills from the pharmacy.

- Goals. They arise when one wants to obtain a state in a property of the environment that with the own capacities is not possible. For example, due to the situation that Juan's pill dispenser is empty, he must make the electronic prescription and also request some doses from his friend Emilio.

- Strategy Dispatcher. Devices can detect what goals there are in the environment, and which ones can be solved with their skills. A strategy is identified when it is detected how to coordinate the 
devices in the environment to solve the given goals. The complexity of strategies lies in the collaboration of entities to identify and solve needs. Returning to the example of Juan and Emilio, Emilio's pill dispenser must establish a strategy to give a few doses to Juan, but only if Emilio has plenty of pills.

- Knowledge Engine. It analyzes the history of the entity's activities to detect patterns and learn from them, with the goal of automating tasks in the future or detect routines.

This architecture achieves the interconnection of IoT devices at the features level. The interconnection is based on relating the skills of one entity with the goals of another. We know that the goals in an entity arise from the lack of skills when obtaining a desired state in the environment, so we must know how to perform this interconnection and that the goals can be resolved in the best way. Each entity has its own vision of the context, and knows the skills and goals of nearby entities, so that it can interact with them. This is achieved by integrating Situational-Context with Semantic Web and ontologies.

By looking that an ontology can represent the skills and goals of Juan's pill dispenser, we know what kind of goals could be covered by this entity. When Juan's pill dispenser detects that he has run out of pills, it interprets its social profile data and, as it knows that Emilio takes the same pills, and it can ask him for a couple of doses. In this way it will be able to automatically use its ability to cover the person need. The Semantic Web will be responsible for providing knowledge to this information, making the relationships between skills and goals are defined in a more human language, and also interpretable by machines.

\begin{tabular}{|l|l|}
\hline \multicolumn{1}{|c|}{ Personal info } & \multicolumn{1}{c|}{ Skills } \\
\hline Manufacturer & Pills management \\
\hline - PDOne & - Reminder doses \\
\hline Model & - Distribute \\
\hline - One & - Recommendations \\
\hline Family & $-\ldots$ \\
\hline - HealthCare & Pharmacy \\
\hline Device & - Request pills \\
\hline - Pill dispenser & - Electronic receipt \\
\hline - ... & $-\ldots$ \\
\hline
\end{tabular}

\begin{tabular}{|l|l|}
\hline \multicolumn{1}{|c|}{ Personal info } & \multicolumn{1}{c|}{ Goals } \\
\hline Manufacturer & Pills management \\
\hline - Huawei & - Reminder doses \\
\hline Model & - Check dose amount \\
\hline - P20 Lite & $-\ldots$ \\
\hline Family & Security \\
\hline - Smartphone & - Family notify \\
\hline$-\ldots$ & $-\ldots$ \\
\hline
\end{tabular}

Table 2. Juan

Table 1. Juan's pill dispenser

To better explain the composition of the entities used in our scenario, some of the information stored in their virtual profiles is specified in Tables 1 and 2, where we can see Juan's pill dispenser and Juan, represented through his smartphone. Apart from the personal information, we observe that Juan's pill dispenser has a series of skills with which it can solve Juan's needs.

Based on the information coming from the virtual profile, each entity builds its own ontology. The purpose of this ontology is to relate the discovered entities through their skills and goals. The resolution of the goals of a given entity is to find a skill that is capable of solving it adequately. This mapping is done through simple queries with SPARQL. SPARQL is a RDF (Resource Description Framework) query language and a W3C recommendation (Sirin \& Parsia, 2007). RDF is a standard model for data exchange on the Web and is widely used as an organization scheme for ontologies (Lassila \& Swick, 1999). Therefore, SPARQL contains capabilities to query the necessary and optional information from ontologies, and among other features, to identify which skill should be used to solve a given goal. This search is defined in a preliminary way through the name of the skill and goal. We are currently working on evolving the composition of these queries to make them more precise and complex. An example is shown below in Table 3 . 


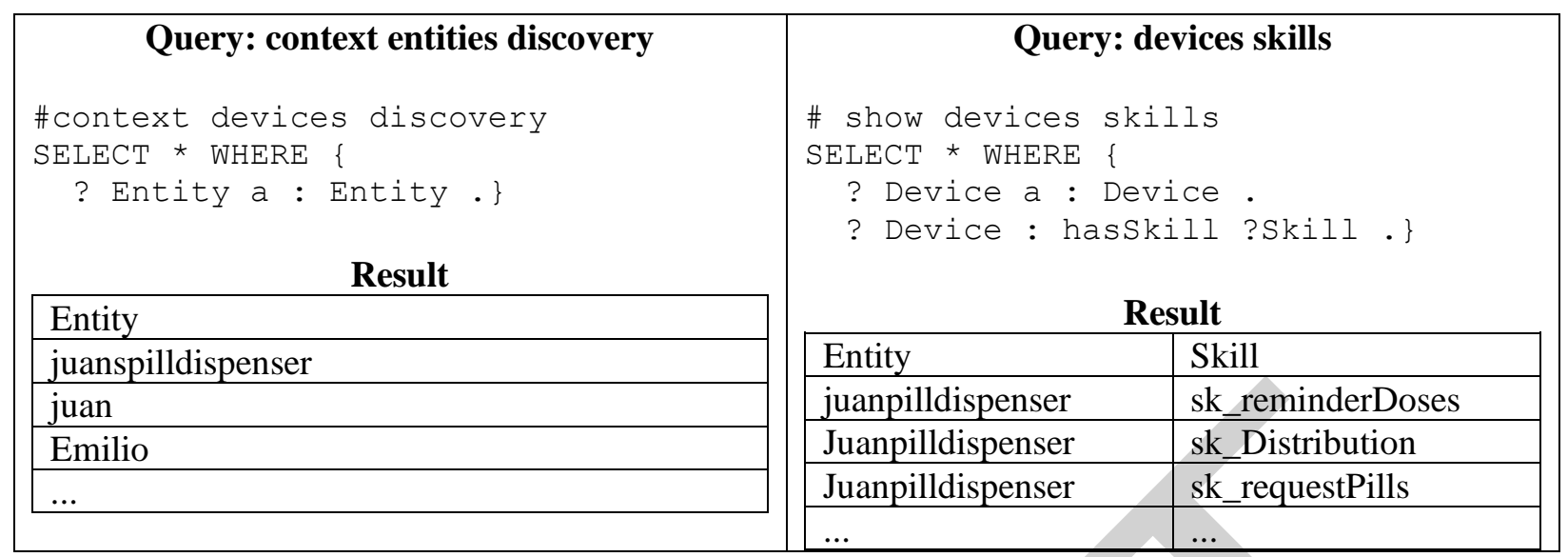

Table 3. SPARQL query example

In our example, Juan needs to take his daily dose according to the medical prescription that his smartphone stores, but due to the lack of dose in the pill dispenser he cannot do it. In this case, thanks to the virtual profiles generated through Situational-Context, Juan knows that Emilio is taking the same medication and he can request him to send several doses. In addition, the interconnection of these entities, people and devices, is done in a totally dynamic way at runtime, so no previous configuration or manual action is necessary, which allows us to improve the people QoL by not having to be aware of the devices or having to invest our valuable time in configure them manually.

Virtual profiles are developed following the PeaaS (People as a Service) paradigm (Guillen et al., 2014) and using the novel tool developed by Tim Berners-Lee's team: Solid (Social Linked Data) (Mansour et al., 2016). Solid proposes a decentralized platform for social web applications, where user data is managed independently of the applications that consume this data, a proposal that is quite aligned with our work. Solid is a framework that can be used to implement the basic pillars of PeaaS, obtaining the following benefits (Berners-Lee, 2017): (1) True data ownership, where users should have the freedom to choose where their data resides and who is allowed to access it; (2) Modular design, because applications are decoupled from the data they produce, users will be able to avoid vendor lock-in, seamlessly switching between apps and personal data storage servers; and (3)Reusing existing data, developers will be able to easily innovate by creating new apps or improving current apps, all while reusing existing data that was created by other apps. This could be a good approach to build part of Situational-Context paradigm and could come in handful of benefits.

\section{INTERCONNECTION FLOW}

The devices interconnection in Situational-Context has a clear objective: the needs resolution. As we mentioned below, an entity has a need when its skills cannot achieve the desired state in the environment and it must draw on the skills of another one.

Through the Connectivity Manager, entities can connect to each other, and exchange information. For example, Juan's pill dispenser and Juan's smartphone can connect each other through Bluetooth.

This information is interpreted and updated by the Context Manager. At this point, the entity knows its own skills and goals, as well as those of the other entities in the context, and will know if it can solve any determined goal. Juan's pill dispenser knows that Juan does not have pills and that there are other elements in the environment with the skill to provide them, such as the pharmacy or Emilio's pill dispenser. 
Then, Juan's smartphone will detect the information coming from the Profile, which contains the entity history and preferences that has a goal. Thanks to this information, Juan is reminded to have his doses by his pill dispenser every day.

Once the change to be made is detected, for example, to give a couple of doses to Juan, the Strategy Dispatcher will formulate a strategy and propose a change over the context. This strategy will consider the skills of the entity and also those of others that could contribute to solving the goal. Using an ontology, the strategy knows which skills and goals are related to each other. Once the strategy has been formulated it is carried out. Besides, it is stored in the profile in order to be able to infer on it and detect possible patterns, to facilitate the strategy development in the future. This whole process is carried out at runtime, without predefining the previous behaviour of the entities, as the solutions to be explored would be innumerable.

Figure 5 shows the interconnection diagram between the entities in the scenario.

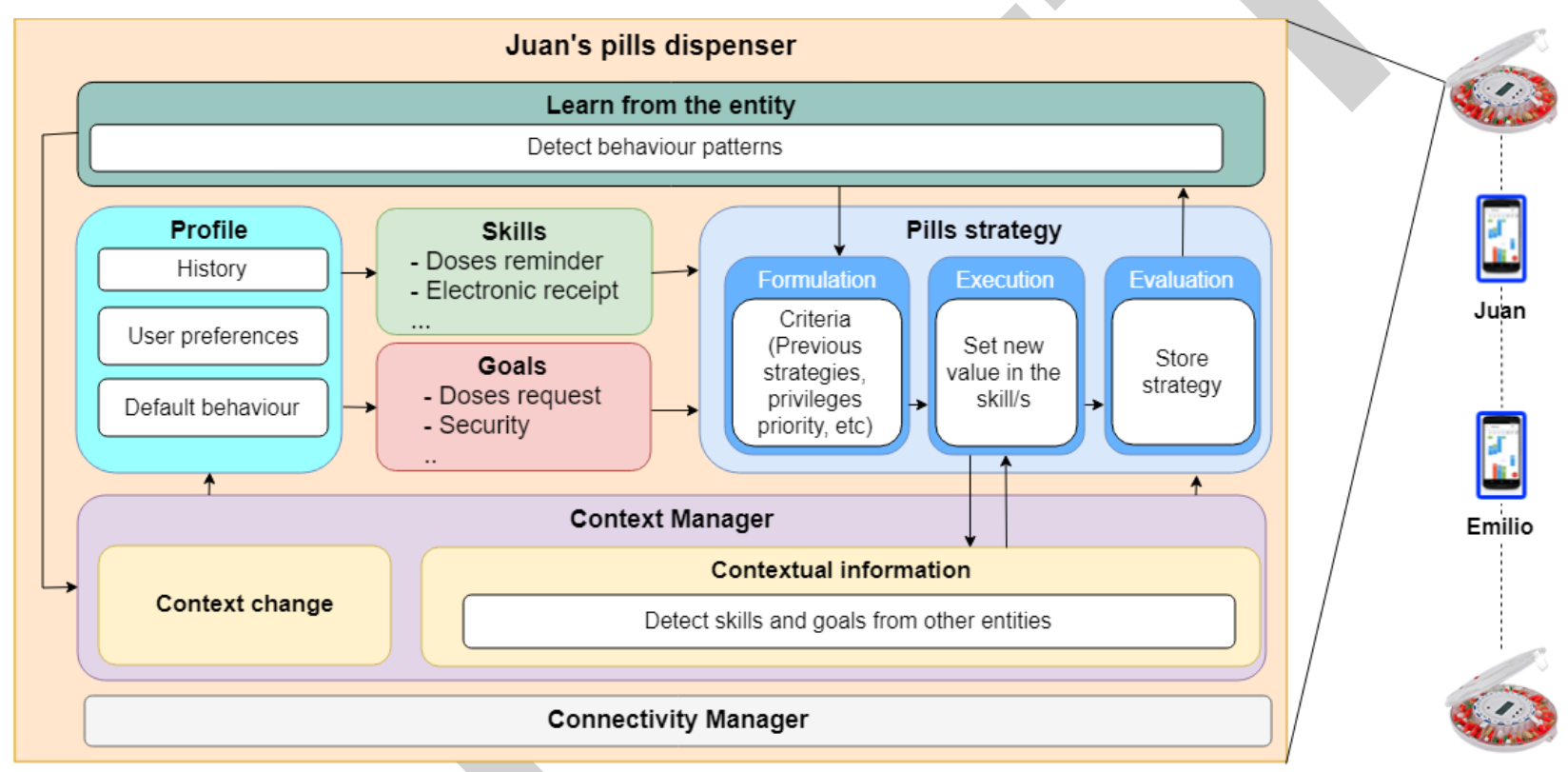

Figure 5. Interconnection between entities

For the specific case of the strategy of pills in the context of Juan, the pill dispenser takes into account the preferences of Juan and Emilio to distribute the pills so that Juan can receive his doses and Emilio has enough pills for the rest of the days. Figure 6 shows the components of the architecture defined above from the point of view of the communication flow for this particular situation. 


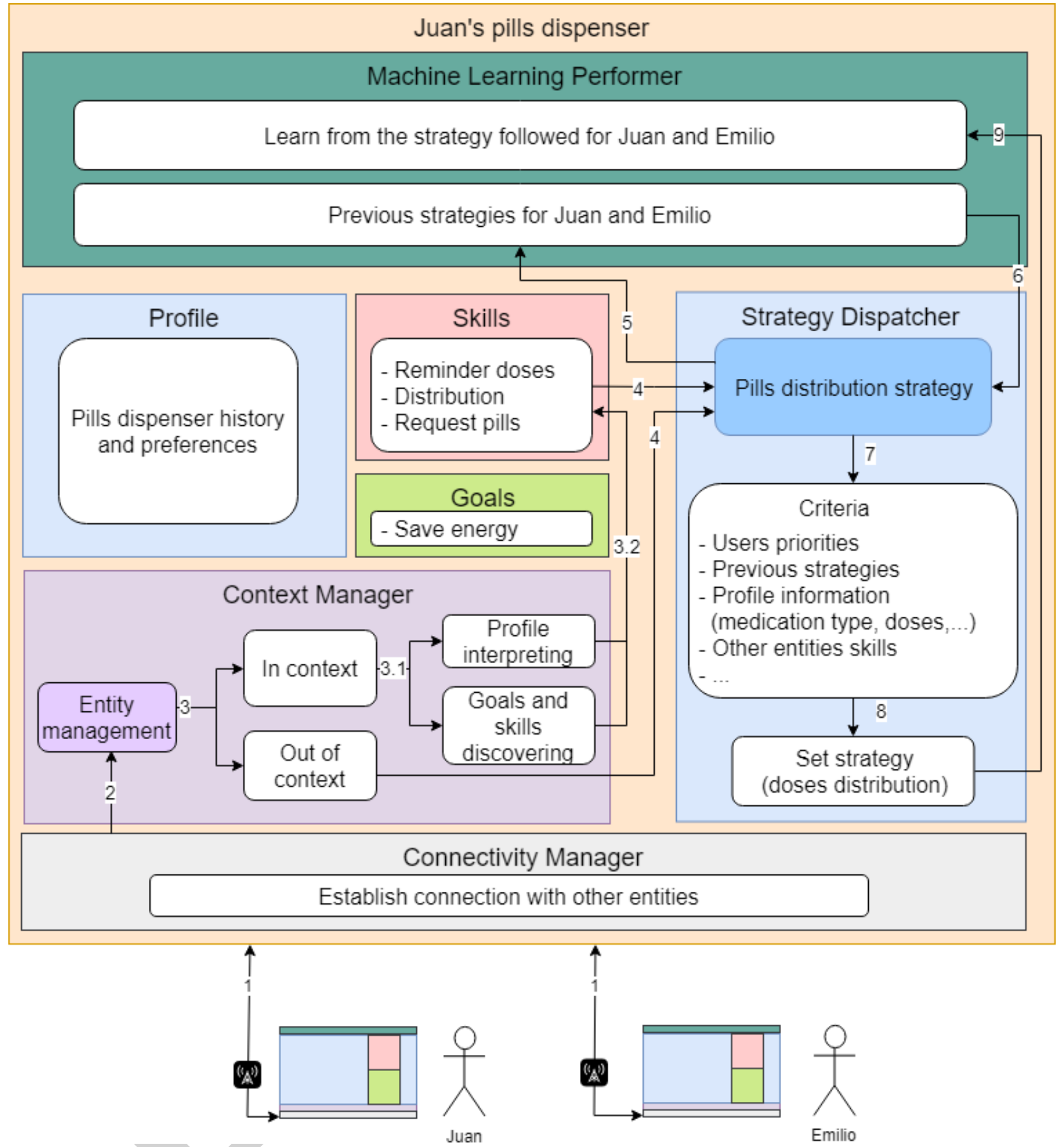

Figure 6. Communication flow for medication distribution

These strategies take into account other issues such as previous strategies, user privileges or their priority, when solving a need. Not all entities would have the same permissions within the context. We differentiate between the entity owning the context, and invited entities, and within these, we have several levels of privileges depending on parameters such as age within the context or level of importance. In this way, when it comes to covering a common need for several entities, those entities with more privileges are favoured, but also fulfilling the needs of the others. As the pillbox belongs to Juan, it must first satisfy his needs before those of the others. For example, if there were only a few doses left for him, none would be given to Emilio. 
The execution of these strategies produces a change in the current context, modifying the behaviour of some entities and, in addition, learns which are the preferences of the entity or entities that are involved, so that, in future interactions, these strategies can be executed more precisely.

Similarly, the rest of entities such as Emilio's pill dispenser, or Juan and Emilio themselves, would have a representation similar to that of Juan's pill dispenser, and with identical behaviour.

\section{RELATED WORK}

As we discussed at the beginning of this research in (Flores-Martin, 2017), we can use different paradigms such as AmI, COP, SW and ML, to automate interactions between users and IoT systems according to user preferences. In addition, solutions to improve integration between people and IoT systems through the use of smartphones such as People as a Service (PeasS) and Internet of People (IoP) were also discussed.

When we delve into Semantic Web aspects within the scope of IoT, we find several works that follow an objective similar to ours. SocioTal (Bernal Bernabé et al., 2017) is a project focused mainly on issues of security and data sharing, whose aim is to create a configurable and secure IoT environment that encourages people to contribute with their devices and information, providing appropriate tools and mechanisms that simplify complexity and encourage citizen participation. Gyrard et al. also address issues related to IoT and Semantic Web, and they even have developed their own framework to facilitate interaction between IoT devices from a template generator for different IoT domains (Gyrard, Datta, Bonnet, \& Boudaoud, 2015), based on Semantic Web technologies to explicitly describe the meaning of sensor measurements. In (Datta, Bonnet, Gyrard, Da Costa, \& Boudaoud, 2015), an architecture for personalized medical care in intelligent homes that allows continuous monitoring of physical parameters and processing of medical data is presented, where Semantic Web technologies are used to combine sensor data from different domains allowing interaction between heterogeneous devices.

As mentioned in Section 1, healthcare domain is gaining great importance within the IoT. We can find works focused on the care or treatment of the elderly. Yuehong et al. (Yuehong, Zeng, Chen, \& Fan, 2016) provide an overview of IoT applications in the healthcare industry, based on a comprehensive literature review and discussion of researchers' achievements. This work is carried out from the perspectives of enabled technologies and methodologies, intelligent devices and systems based on IoT and the several applications of IoT in the healthcare industries. In (Pinto, Cabral, \& Gomes, 2017), we find We-care, a system for the assistance of elderly people that is able to monitor and record the vital information of these people, as well as provide mechanisms to activate alarms in emergency situations. Along the same lines, Mainetti et al. (Mainetti, Patrono, Secco, \& Sergi, 2016) have designed an Ambient System Living (AAL) system to create better living conditions for older people, capable of constantly monitoring their state of health through data from heterogeneous sources. Moreover, many IoT-based patient monitoring systems have a gateway between a sensor network and the Internet. In this sense, Rahmani et al. (Rahmani et al., 2015) propose a system called Smart e-Health Gateway, which assumes responsibility for managing the load of the sensor network and a remote health care center. This development addresses many challenges in ubiquitous health care systems, such as energy efficiency, scalability, and ubiquitous health monitoring and reliability issues.

In addition, if we combine Semantic Web with elderly care, we find an interesting project, SOPRANO (Wolf, Schmidt, \& Klein, 2008), an extensible and open AAL platform for elderly people that aims to lead a more independent life in their family environment through a new generation of intelligent home with ambient intelligence. 
We are aware that there are many proposals for the development of software whose behaviour adapts to the context, but that, to the best of our knowledge, do not cover in many cases the problems mentioned above, such as those related to the adaptation of devices to the conditions of the context at runtime. Therefore, the research challenges we address are several. First, the lack of a unified model of human-IoT interaction. IoT devices are produced by several manufacturers, each with its own interaction model. Secondly, the lack of an automatic negotiation model for the interaction between people and IoT devices according to people's preferences. Some of the works mentioned above pursue a similar goal to ours, in terms of achieving an adaptive context in IoT, but we want to make the interconnection of these IoT devices emerge from the situation itself. If these problems could be solved, there would be a better integration of people in IoT environments in terms of interoperability.

\section{CONCLUSIONS}

As the health care domain is becoming increasingly important, we are concerned about the idea of being able to connect as many smart devices as possible to make the lives of elderly people easier. However, the problem of interconnection in the IoT world is still present today due to the heterogeneity of devices on the market. The interaction between IoT devices is crucial for the resolution of strategies to support people daily tasks and must allow them to adapt their behaviours to people's needs, which often depends on the collaboration of several smart devices.

This work is another step towards achieving this interconnection in a dynamic way, thanks to technologies such as Situational-Context. Thus, we can adapt the behaviour of smart devices to the needs of people in real time, without the need to attend to previous configurations in design time.

\section{ACKNOWLEDGMENT}

This work was supported by the European Regional Development Fund (ERDF) and by 4IE project (00454IE-4-P) funded by the Interreg V-A España-Portugal (POCTEP) 2014-2020 program, by the Spanish Ministry of Science and Innovation through project RTI2018-094591-B-I00 (MINECO/FEDER, UE) and by the Department of Economy and Infrastructure of the Government of Extremadura (GR18112, IB18030).

\section{REFERENCES}

Barnaghi, P., Wang, W., Henson, C., \& Taylor, K. (2012). Semantics for the Internet of Things: early progress and back to the future. International Journal on Semantic Web and Information Systems (IJSWIS), $8(1), 1-21$.

Bernabe, J. B., Elicegui, I., Gandrille, E., Gligoric, N., Gluhak, A., Hennebert, C., ... \& Nati, M. (2017, June). SocIoTal-The development and architecture of a social IoT framework. In 2017 Global Internet of Things Summit (GIoTS) (pp. 1-6). IEEE.

Berners-Lee, T. (2017). Solid vision. https://solid.mit.edu/. (Accessed on 2018-10-09)

Berrocal, J., Garcia-Alonso, J., Canal, C., \& Murillo, J. M. (2016, June). Situational-Context: a unified view of everything involved at a particular situation. In International Conference on Web Engineering (pp. 476483). Springer, Cham.

Datta, S. K., Bonnet, C., Gyrard, A., Da Costa, R. P. F., \& Boudaoud, K. (2015, October). Applying Internet of Things for personalized healthcare in smart homes. In 2015 24th Wireless and Optical Communication Conference (WOCC) (pp. 164-169). IEEE.

Flores-Martin, D. (2017, November). Meeting IoT Users' Preferences by Emerging Behavior at Run-Time. In International Conference on Service-Oriented Computing (pp. 333-338). Springer, Cham.

Galán-Jiménez, J., Berrocal, J., Garcia-Alonso, J., Canal-Velasco, J. C., \& Murillo, J. M. (2017). Coordinating heterogeneous IoT devices by means of the centralized vision of the SDN controller.

Guillen, J., Miranda, J., Berrocal, J., Garcia-Alonso, J., Murillo, J. M., \& Canal, C. (2013). People as a service: a mobile-centric model for providing collective sociological profiles. IEEE software, 31(2), 48-53. 
Gyrard, A., Datta, S. K., Bonnet, C., \& Boudaoud, K. (2015, August). Cross-domain Internet of Things application development: M3 framework and evaluation. In 2015 3rd International Conference on Future Internet of Things and Cloud (pp. 9-16). IEEE.

Haluza, D., \& Jungwirth, D. (2015). ICT and the future of health care: aspects of health promotion. International journal of medical informatics, 84(1), 48-57.

IronPaper. (2015). Ironpaper. https://www.ironpaper.com/webintel/articles/internet-things-marketstatistics-2015/. (Accessed on 2019-04-26)

Kim, J., Yun, J., Choi, S. C., Seed, D. N., Lu, G., Bauer, M., ... \& Song, J. (2016). Standard-based IoT platforms interworking: implementation, experiences, and lessons learned. IEEE Communications Magazine, 54(7), 48-54.

Lassila, O., \& Swick, R. R. (1998). Resource description framework (RDF) model and syntax specification. Mainetti, L., Patrono, L., Secco, A., \& Sergi, I. (2016, July). An IoT-aware AAL system for elderly people. In 2016 International Multidisciplinary Conference on Computer and Energy Science (SpliTech) (pp. 1-6). IEEE.

Mansour, E., Sambra, A. V., Hawke, S., Zereba, M., Capadisli, S., Ghanem, A., ... \& Berners-Lee, T. (2016, April). A demonstration of the solid platform for social web applications. In Proceedings of the 25th International Conference Companion on World Wide Web (pp. 223-226). International World Wide Web Conferences Steering Committee.

Metcalf, D., Milliard, S. T., Gomez, M., \& Schwartz, M. (2016). Wearables and the internet of things for health: Wearable, interconnected devices promise more efficient and comprehensive health care. IEEE pulse, 7(5), 35-39.

Nordrum, A. (2016). The internet of fewer things [news]. IEEE Spectrum, 53 (10), 12-13.

Perera, C., Zaslavsky, A., Christen, P., \& Georgakopoulos, D. (2014). Context Aware Computing for the Internet of Things: A survey. IEEE Communications Surveys \& Tutorials, 16 (1), 414-454.

Pinto, S., Cabral, J., \& Gomes, T. (2017, March). We-care: An IoT-based health care system for elderly people. In 2017 IEEE International Conference on Industrial Technology (ICIT) (pp. 1378-1383). IEEE.

Rahmani, A. M., Thanigaivelan, N. K., Gia, T. N., Granados, J., Negash, B., Liljeberg, P., \& Tenhunen, H. (2015, January). Smart e-health gateway: Bringing intelligence to internet-of-things based ubiquitous healthcare systems. In 2015 12th Annual IEEE Consumer Communications and Networking Conference (CCNC) (pp. 826-834). IEEE.

Roman, R., Zhou, J., \& Lopez, J. (2013). On the features and challenges of security and privacy in distributed internet of things. Computer Networks, 57(10), 2266-2279.

Shrestha, N., Kubler, S., \& Främling, K. (2014, August). Standardized framework for integrating domainspecific applications into the IoT. In 2014 International Conference on Future Internet of Things and Cloud (pp. 124-131). IEEE.

Sirin, E., \& Parsia, B. (2007, June). SPARQL-DL: SPARQL Query for OWL-DL. In OWLED (Vol. 258). Swetina, J., Lu, G., Jacobs, P., Ennesser, F., \& Song, J. (2014). Toward a standardized common M2M service layer platform: Introduction to oneM2M. IEEE Wireless Communications, 21(3), 20-26.

Szilagyi, I., \& Wira, P. (2016, October). Ontologies and Semantic Web for the Internet of Things-a survey. In IECON 2016-42nd Annual Conference of the IEEE Industrial Electronics Society (pp. 6949-6954). IEEE.

Taivalsaari, A., \& Mikkonen, T. (2017). A roadmap to the programmable world: software challenges in the IoT era. IEEE Software, 34(1), 72-80.

Wolf, P., Schmidt, A., \& Klein, M. (2008, July). SOPRANO-An extensible, open AAL platform for elderly people based on semantical contracts. In 3rd Workshop on Artificial Intelligence Techniques for Ambient Intelligence (AITAmI'08), 18th European Conference on Artificial Intelligence (ECAI 08), Patras, Greece. Yuehong, Y. I. N., Zeng, Y., Chen, X., \& Fan, Y. (2016). The internet of things in healthcare: An overview. Journal of Industrial Information Integration, 1, 3-13. 